\title{
Mouse Odf2 localizes to centrosomes and basal bodies in adult tissues and to the photoreceptor primary cilium
}

\author{
Stephanie Schweizer • Sigrid Hoyer-Fender
}

Received: 8 May 2009 /Accepted: 12 August 2009 /Published online: 10 September 2009

(C) The Author(s) 2009. This article is published with open access at Springerlink.com

\begin{abstract}
Odf2 (outer dense fiber 2) is the major protein of the cytoskeleton of the sperm tail. In somatic cells, it is a component of the centrosome in which it is located in the appendages of the mother centriole. Additionally, as shown previously by forced expression in cultured cells, Odf2 localizes to centrioles, basal bodies, and primary cilia, which are all structurally and functionally interconnected. The importance of Odf2 has become obvious by the absence of primary cilia in Odf2-deficient cells and by the embryonic lethality of the Odf2 gene trap insertional mouse. However, nothing is known about the endogenous localization of Odf2 in the tissues of adult mice. We show here that Odf2 protein localizes to centrosomes, to photoreceptor primary cilia, and to basal bodies of ciliated cells of the respiratory epithelium and of the kidney. Our results thus suggest that Odf2 contributes to assorted ciliopathies.
\end{abstract}

Keywords Odf2 · Adult tissues · Photoreceptor cilium · Basal bodies $\cdot$ Mouse (C57BL/6; CD1)

\section{Introduction}

Odf2 (outer dense fiber 2) was first described as a major protein component of the accessory outer dense fibers of

This work was supported by the Deutsche Forschungsgemeinschaft (Ho 1440/3-6).

S. Schweizer $\cdot$ S. Hoyer-Fender $(\bowtie)$

Johann Friedrich Blumenbach Institute of Zoology

and Anthropology - Developmental Biology, GZMB,

Georg August University of Göttingen,

Justus-von-Liebig-Weg 11,

37077 Göttingen, Germany

e-mail: shoyer@gwdg.de the mammalian sperm tail (Brohmann et al. 1997; HoyerFender et al. 1998) in which it localizes to the medulla and cortex and to the connecting piece (Schalles et al. 1998). In situ hybridization and developmental Northern blots have demonstrated that Odf2 expression is initiated after the second meiotic division, starting in round spermatids (Hoyer-Fender et al. 1998), and is also present in the cytoplasm of elongated spermatids. However, Odf2 expression is not restricted to male germ cells but is also found in somatic cells in which the protein is a component of the centrosome, the major microtubule organizing center (MTOC) of the cell (Nakagawa et al. 2001).

The centrosome, as the main MTOC of interphase cells of animals, functions in the polarized outgrowth of microtubules that are themselves central for organizing cell structure, determining cell polarity, and directing cell movement during interphase and for mitotic and meiotic chromosome separation. Most organelles are guided and positioned by microtubules. Hence, the centrosome is vitally important for the cell. In addition, the centrosome seems to play an important role in orchestrating cell cycle progression. In animal cells, the centrosome is composed of a pair of centrioles surrounded by an electron-dense cloud of pericentriolar material, the pericentriolar matrix (Bornens 2002). The two centrioles of a single centrosome are unequal with respect to both their date of origin and their composition. The older or mother centriole is characterized by the presence of distal and subdistal appendages that contain Odf2 and Cenexin (Lange and Gull 1995; Nakagawa et al. 2001), the later an eponym for the older centriole. Cenexin and Odf2 have turned out to be alternative splice variants of one gene (Hüber and Hoyer-Fender 2007; Hüber et al. 2008).

The establishment of the polarity and differentiation of a cell is often associated with the generation of a primary 
cilium. Primary cilia project from the cellular surface and are anchored to the cell by the basal body, which itself has developed from the mother centriole of the centrosome. Primary cilia are mostly immotile with a $9+0$ microtubule arrangement (nine peripheral doublet microtubules without the two central singlet microtubules found in motile cilia) and are found singly on cells. Most epithelial and stromal cells throughout the mammalian body bear primary cilia. As primary cilia project from the cellular surface, they are predestined to sense the cellular environment (Michaud and Yoder 2006). Primary cilia are hence essential sensory organelles, with detrimental effects occurring in the whole body when ciliary function is impaired. The view that all three organelles, viz., the centrosome/centriole, the basal body, and the primary cilium, are structurally and functionally interconnected is strengthened by the finding that certain proteins associated with cilia-related diseases are localized to all three organelles (Badano et al. 2006).

Testicular Odf2 is the major protein of the accessory or outer dense fibers (ODFs) of the sperm tail. Although the ODFs seem not to be involved in active motility, they are crucial for the stability and motility of the sperm tail and hence for fertility. Moreover, in addition to the spermspecific relevance of Odf2, recent identification of Odf2 as a centrosomal component has turned attention to its centrosomal function. Odf2 proteins are coiled-coil proteins that associate with the microtubular cytoskeleton, although they do not bind directly to tubulin (Donkor et al. 2004). Furthermore, the Odf2 variant Cenexin seems to be associated with acetylated microtubules, which are themselves typical for stable microtubules found in centrioles, primary cilia, and axonemata (Hüber et al. 2008). The centrosomal localization of Odf2 implies its involvement in cilia/flagella generation. Indeed, the depletion of Odf2 results in mother centrioles that lack their appendages. These centrioles are unable to generate primary cilia (Ishikawa et al. 2005) denoting the significance of Odf2 in cilia formation. Moreover, mice homozygous for a gene trap insertion into exon 9 of the Odf2 gene, which results in a truncated Odf2 protein, display pre-implantation lethality (Salmon et al. 2006). These results strongly imply that Odf2 is an absolute requirement for embryonic development to proceed and indicate the importance of the integrity of cellular components as centrioles, basal bodies, and/or primary cilia.

Transcription of $O d f 2$ has been reported to proceed throughout somatic tissues (Hüber and Hoyer-Fender 2007) and has also been found at the blastocyst stage (Salmon et al. 2006). We have determined here whether endogenous Odf2 protein can be identified in tissue sections prepared from adult mice and, if so, its localization. We have identified Odf2 on cerebellum sections and on sections through sarcomeres in the close vicinity to nuclei, consistent with centromeric localization. Additionally, Odf2 is found in the photoreceptor cell layer of retinal cross sections and in the photoreceptor primary cilium. Finally, immunocytological inspection of cultivated cells generated from the respiratory epithelium of mice has unequivocally confirmed endogenous Odf 2 as a protein of the basal body. Our results thus support the idea that Odf2 is a centrosomal and basal body protein that is additionally a component of modified photoreceptor primary cilia. Taken together with its reported necessity for the generation of primary cilia (Ishikawa et al. 2005), the embryonic lethality observed in homozygous $\operatorname{Odf} 2$ gene trap insertion mice (Salmon et al. 2006) might be the result of the impairment of primary cilia.

\section{Materials and methods}

Tissue sectioning and immunohistology

Mice were maintained in a temperature- and lightcontrolled room. For all experiments, wild-type mice (C57BL/6 or CD1 strain) were used. Tissues of adult mice were excised and shock-frozen in liquid nitrogen. Tissues were processed for the preparation of cryosections, which were mounted on super-frost slides, fixed in methanol at $-20^{\circ} \mathrm{C}$, and air-dried. Specimens were preincubated in $0.2 \%$ Triton X-100 in PBS (phosphatebuffered saline at $\mathrm{pH} 7.5$ ) for $5 \mathrm{~min}$, washed in PBS, and incubated in $0.2 \%$ bovine serum albumin, $0.1 \%$ Triton X-100 in PBS for the blocking of unspecific binding sites. Antibodies were diluted 1:100 in PBS, and incubation was performed at $37^{\circ} \mathrm{C}$ for $1 \mathrm{~h}$. Thereafter, probes were washed in PBS (three times, $5 \mathrm{~min}$ each), followed by incubation with the secondary antibody. Anti-ODF2-antibody was generated and affinity purified as described (Brohmann et al. 1997). Monoclonal anti- $\gamma$-tubulin (GTU88), anti-acetylatedtubulin (6-11B-1), and anti-mouse Cy3 antibodies were supplied by Sigma Biosciences, St. Louis. Anti-mouse Alexa-488 was from Molecular Probes (Eugene), and antirabbit $\mathrm{Cy} 3$ antibody was from Amersham. DNA was counterstained with 4,6-diamidino-2-phenylindole (DAPI). Images were taken by confocal microscopy (LSM 510, Zeiss) and processed by using Adobe Photoshop 5.0.

Culturing of nasal epithelial cells and immunocytology

The epithelium of a murine nose was excised, cut into small pieces, and cultivated in DMEM, $10 \%$ fetal bovine serum, $5 \%$ penicillin/streptomycin (all Gibco) at $37^{\circ} \mathrm{C}$ in $5 \% \mathrm{CO}_{2}$. Cells grown on coverslips were fixed in 


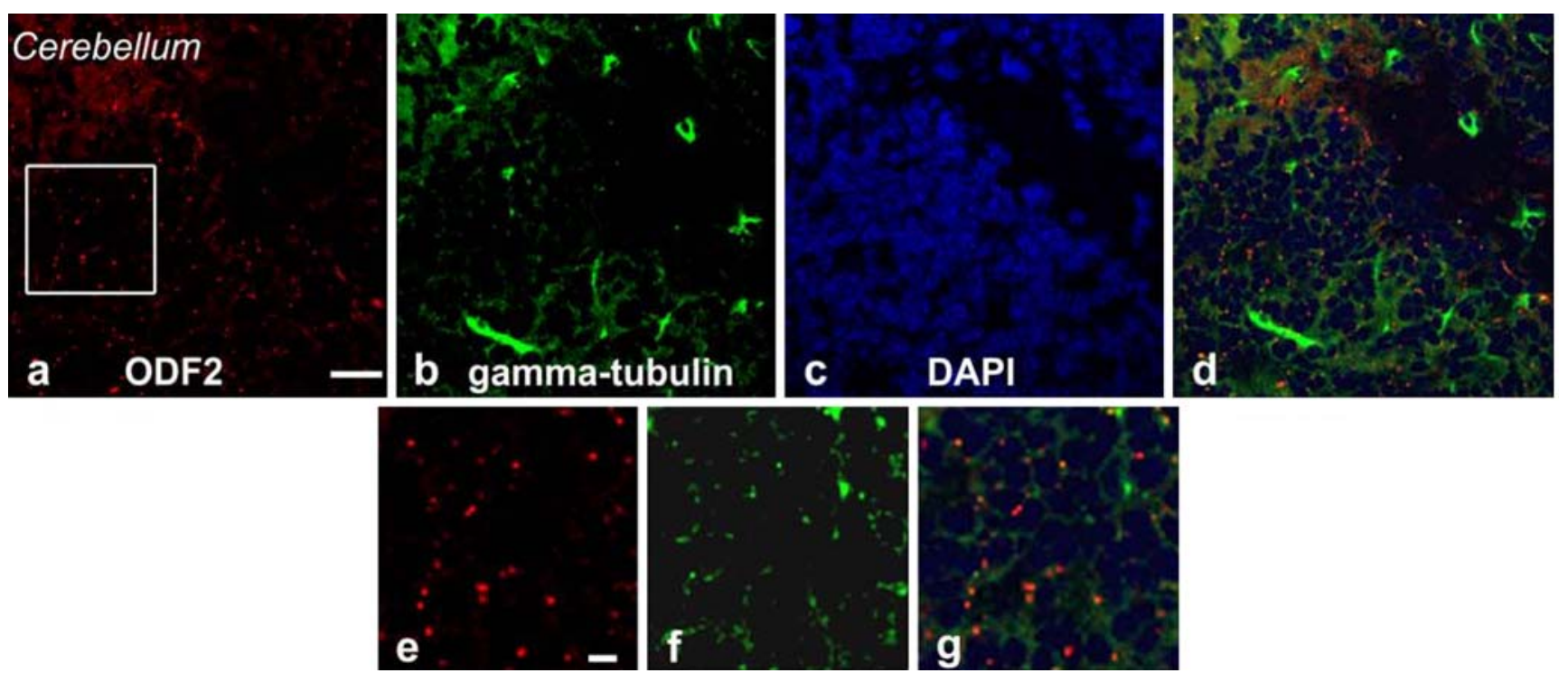

Fig. 1 Punctate staining of Odf2 in cerebellum sections colocalizes with $\gamma$-tubulin, suggesting centrosomal localization. Cerebellum sections of an adult mouse were stained with antibodies against Odf2 (red in $\mathbf{a}, \mathbf{d}, \mathbf{e}, \mathbf{g}$ ) and with $\gamma$-tubulin (green in $\mathbf{b}, \mathbf{d}, \mathbf{f}, \mathbf{g}$ ) as a

$3.7 \%$ paraformaldehyde in $\mathrm{PBS}$ at $\mathrm{pH} 7.5$, preincubated in $0.2 \%$ Triton X-100 in PBS for 5 min, washed in PBS, and subsequently incubated in $0.2 \%$ bovine serum albumin, $0.1 \%$ Triton X-100 in PBS for the blocking of unspecific binding sites. Incubation of the cells on the coverslips with the primary antibodies was performed at $37^{\circ} \mathrm{C}$ for $2 \mathrm{~h}$, followed by repeated washes PBS, and incubation with secondary antibody at $37^{\circ} \mathrm{C}$ for $1 \mathrm{~h}$. DNA was counterstained with DAPI, and images were taken as described. centrosomal marker. Nuclei were counterstained with DAPI (blue in $\mathbf{c}$, $\mathbf{d}, \mathbf{g})$. Merged images are presented in $\mathbf{d}, \mathbf{g}$. High magnifications of the region within the frame depicted in a) are shown in $\mathbf{e}-\mathbf{g}$. Bars $20 \mu \mathrm{m}$ (a), $5 \mu \mathrm{m}(\mathbf{e})$

Isolation of photoreceptor sensory cilia

Photoreceptor sensory cilia (PSC) were isolated essential as described by Liu et al. (2007). In brief, fresh mouse retinas were gently rocked in buffer A (10 mM PIPES, pH 7.0, $5 \mathrm{mM} \mathrm{MgCl}_{2}, 1 \times$ proteinase inhibitor mix) containing $50 \%$ sucrose. PSC complexes were separated by centrifugation for $20 \mathrm{~min}$ at $13,000 \mathrm{~g}$ and thereafter collected from the top layer, diluted 1:1 in buffer A without sucrose, and centrifuged
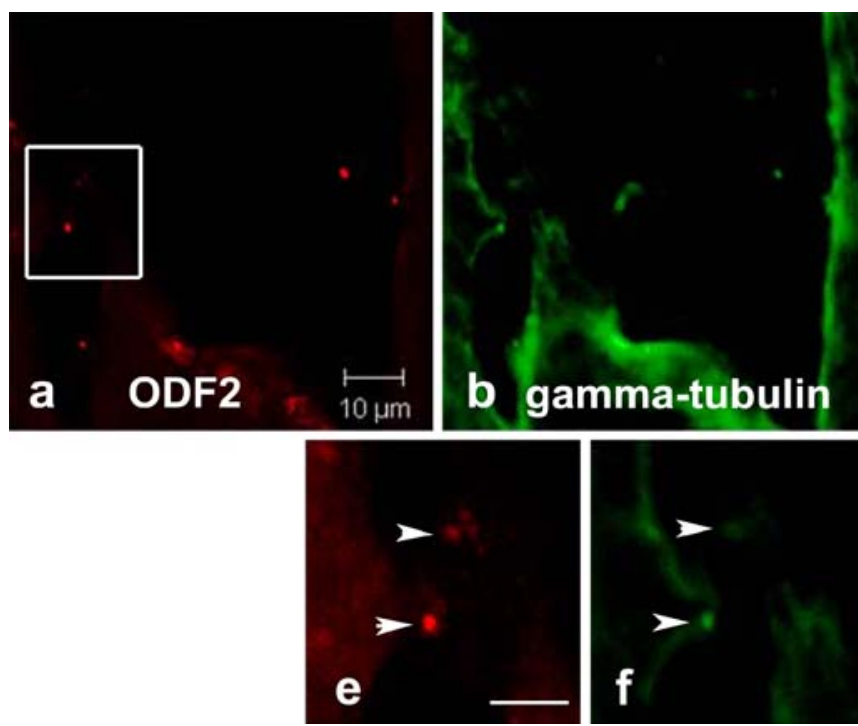

Fig. 2 In muscle tissue, Odf2 colocalizes with $\gamma$-tubulin at the nuclear periphery, thus demonstrating a centrosomal localization. Cross sections of sarcomeres were stained with antibodies against Odf2 (red in $\mathbf{a}, \mathbf{d}, \mathbf{e}, \mathbf{h}$ ) and against $\gamma$-tubulin (green in $\mathbf{b}, \mathbf{d}, \mathbf{f}, \mathbf{h}$ ) as a

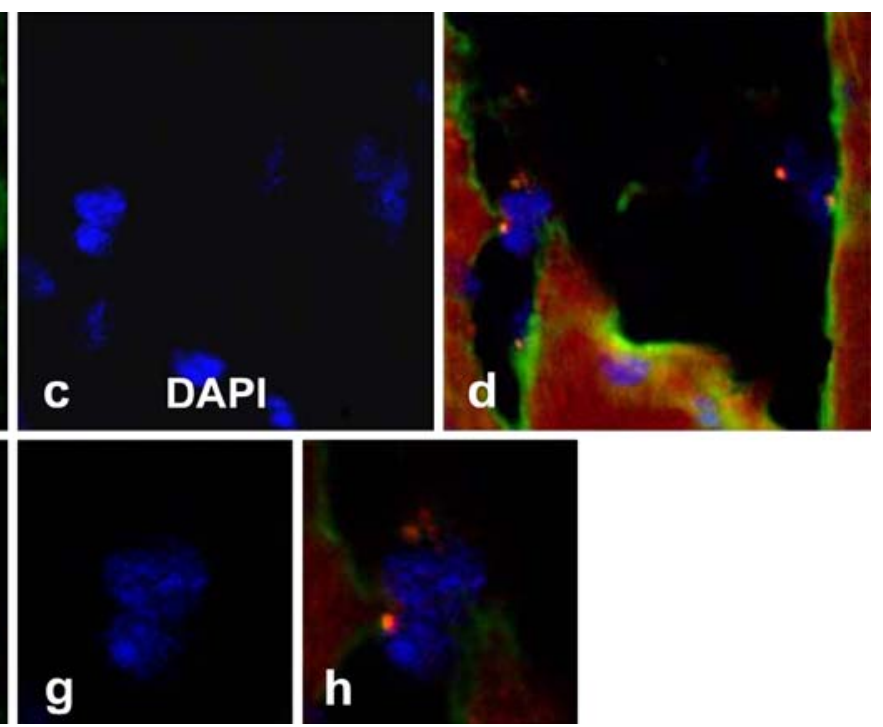

centrosomal marker. Nuclei were counterstained with DAPI (blue in $\mathbf{c}$, $\mathbf{d}, \mathbf{g}, \mathbf{h})$. Merged images are presented in $\mathbf{d}, \mathbf{h}$. High magnifications of the region within the frame depicted in $\mathbf{a}$ are shown in $\mathbf{e}-\mathbf{h}$ (arrowheads centrosomes). Bar in e $2.5 \mu \mathrm{m}$ 
Fig. 3 Odf2 (green in b) is found at the outer border of the outer nuclear layer $(O N L)$ in a punctate pattern colocalizing with $\gamma$-tubulin (red in a). Additionally, Odf2 weakly stains the inner segment $(I S)$ of photoreceptor cells ( $O S$ outer segment). Nuclei were counterstained with DAPI (blue in c). Merged image is presented in d. Bar $20 \mu \mathrm{m}$
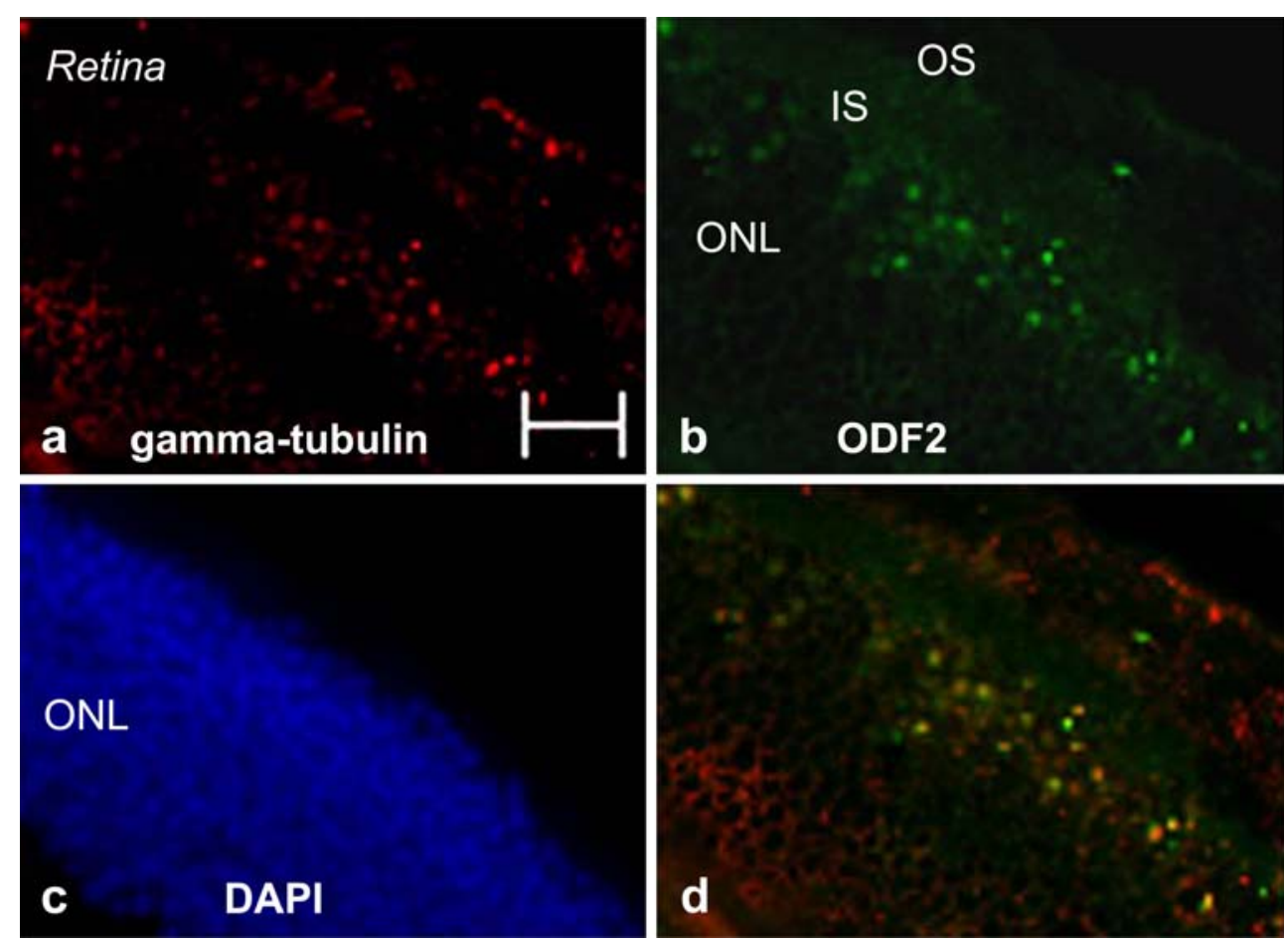

again on a $50 \%$ sucrose cushion. The complexes, which were then found at the interface, were collected, sedimented by decreasing the sucrose concentration, fixed in $3.7 \%$ paraformaldehyde, and transferred to slides. Immunocyotology was performed as described.

\section{Results and discussion}

Endogenous Odf2 localizes to centrosomes in sections of brain and muscle tissue

The subcellular localization of Odf2 has been mainly investigated in cultured cells by means of forced Odf2 fusion protein expression (Hüber et al 2008; Soung et al. 2006). This approach has shown that Odf2 is associated with acetylated microtubules (Hüber et al. 2008) and that it localizes to the centrosome, the basal body, and the primary cilium. Additionally, endogenous Odf2 has been detected in cultured cells in the centrosome and the microtubular spindle poles (Nakagawa et al. 2001) as well.

Odf2 is ubiquitously transcribed in somatic tissues (Hüber and Hoyer-Fender 2007). However, its localization in tissues has never previously been investigated. We have focused here on brain tissue and on muscle tissue, the later as an example for those tissues in which primary cilia have not previously been investigated, and on tissues that contain ciliated cells (kidney, retina, nasal epithelial cells). The antibody used was
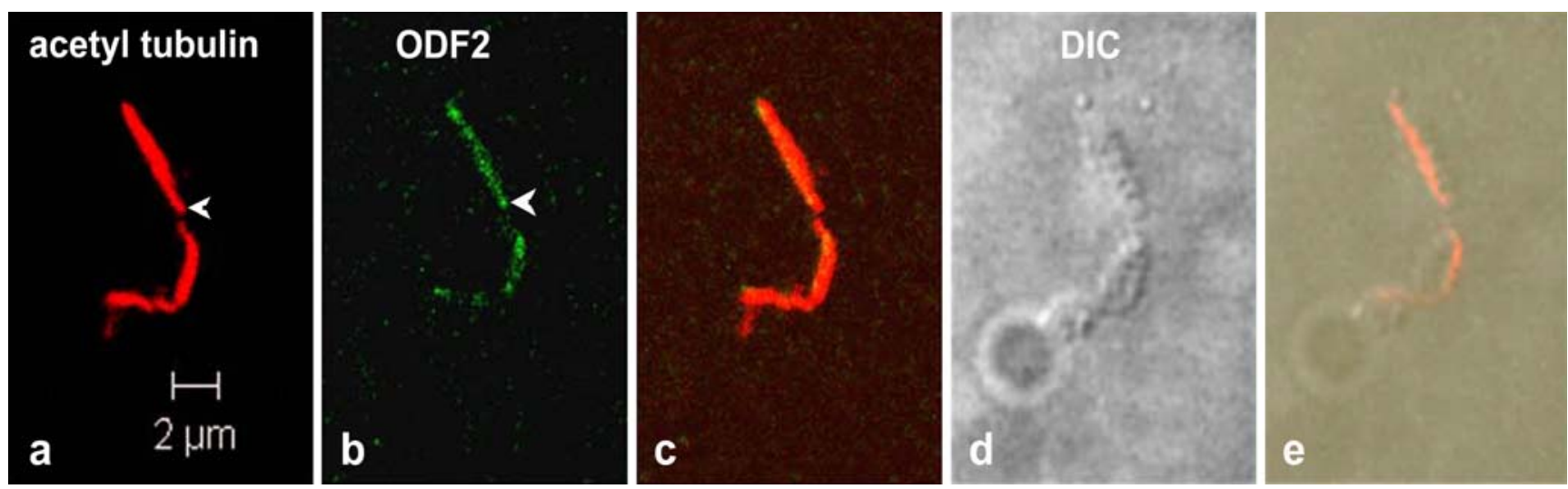

Fig. 4 Immunostaining of isolated photoreceptor sensory cilia (PSC). Odf2 localizes to the primary cilium of photoreceptor outer segments and marks the connecting cilium (arrowheads) as a dot (b). Staining for acetylated tubulin in red (a), antibody staining for Odf 2 in green

(b), and merged image (c). The differential interference contrast (DIC) image is shown in $\mathbf{d}$. The merged image of the DIC and fluorescence images is presented in $\mathbf{e}$ 


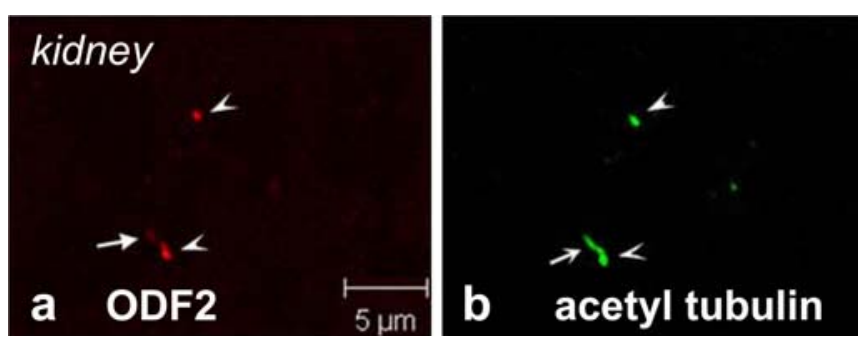

Fig. 5 Odf2 localizes to the base of primary cilia in mouse kidney cross sections. Primary cilia (arrows) and basal bodies (arrowheads) are immunocytologically stained with antibodies against acetylated

generated against the C-terminal region of testicular Odf2, which is present in all Odf2 isoforms reported to date. Our results thus demonstrate the distribution of endogenous Odf2 irrespective of which alternatively spliced isoform is present. In cerebellar tissue sections, Odf2 is found in a punctate pattern at the nuclear periphery (Fig. 1), a pattern reminiscent of centrosomes. We additionally have used anti $\gamma$-tubulin staining to confirm centrosomal localization (Fig. 1). However, although Odf2 colocalizes with $\gamma$-tubulin spots (Fig. 1e-g; colocalization was found in $84 \%$ of all dots, $n=31$ ), the high $\gamma$-tubulin background staining most likely reflects high amount of cytoplasmic $\gamma$-tubulin. Thus, we conclude that Odf2 most likely localizes to centrosomes in the cerebellum. Additionally, Odf2 seems to be a more suitable centrosome marker in the brain than $\gamma$-tubulin.

In muscle tissue sections, we also found colocalization of endogenous Odf 2 and $\gamma$-tubulin (Fig. 2) at the nuclear periphery, thus supporting once again a centrosomal localization. However, the high background staining of $\gamma$-tubulin and of Odf 2 makes muscle tissue sections difficult to investigate immunologically.

Odf2 is located at the border of the outer nuclear layer and in the photoreceptor inner segment of mouse retina

The vertebrate retina is a light-sensing organ composed mainly of photoreceptor cells and neurons that process

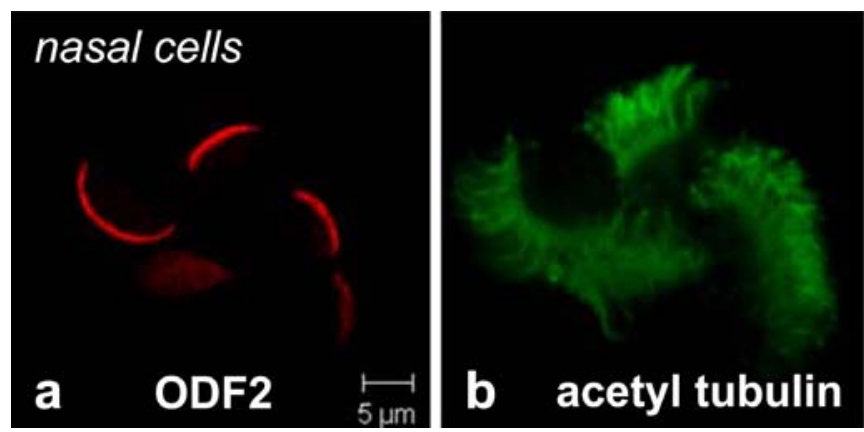

Fig. 6 Odf2 is a basal body protein in nasal epithelial cells. Primary cells of the nasal epithelium of an adult mouse were cultivated on coverslips and processed for antibody staining against Odf2 (red in a) and against acetylated tubulin (green in b). Acetylated tubulin marks
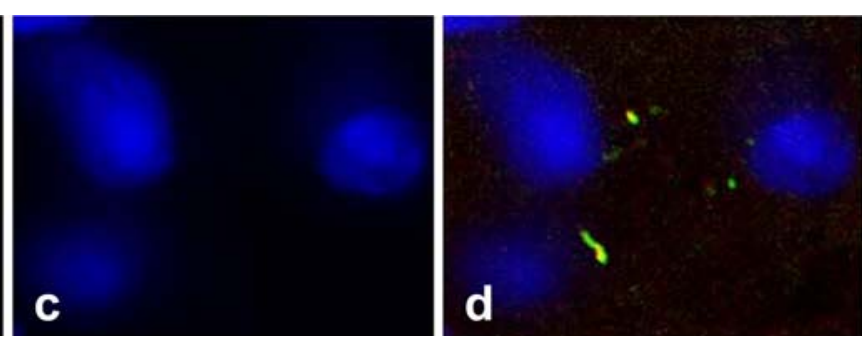

tubulin (green in b). Odf2 antibody staining appears red in a. Nuclei are counterstained with DAPI (c). Merged image is presented in $\mathbf{d}$

the incoming neural signals. The retina is structured into several neuronal layers with the nuclei of photoreceptors being located in the outer nuclear layer (ONL). The light-sensitive outer segments (OS) of the photoreceptor cells are located at the back of the retina. These OSs of retinal rods and cones bear the photoreceptor pigment and are well-known as modified cilia. The PSC are important for the transport and the generation of rhodopsin-bearing disks. Like other cilia, PSC contain an axoneme, which grows out from the basal body, located in the metabolically active inner segment (IS), and passes through a transition zone, also known as the connecting cilium, into the OS. The basal bodies are anchored to the photoreceptor cell bodies by rootlets, which additionally function as a channel for proteins destined for the OS.

Immunocytological staining of mouse retina cryosections with anti-Odf2-antibody revealed intense dotted staining at the outer border of the ONL colocalizing with $\gamma$ tubulin (Fig. 3). Additionally, weak staining was seen at the IS of photoreceptor cells just beneath the ONL (Fig. 3). To confirm Odf2 localization in photoreceptor cells, we isolated PSC complexes from mouse retina and performed immunostaining with antibodies against acetylated tubulin and against Odf2 (Fig. 4).The photoreceptor OS was identified by detecting the primary cilium with antibodies against acetylated tubulin (red in Fig. 4a) and by differential

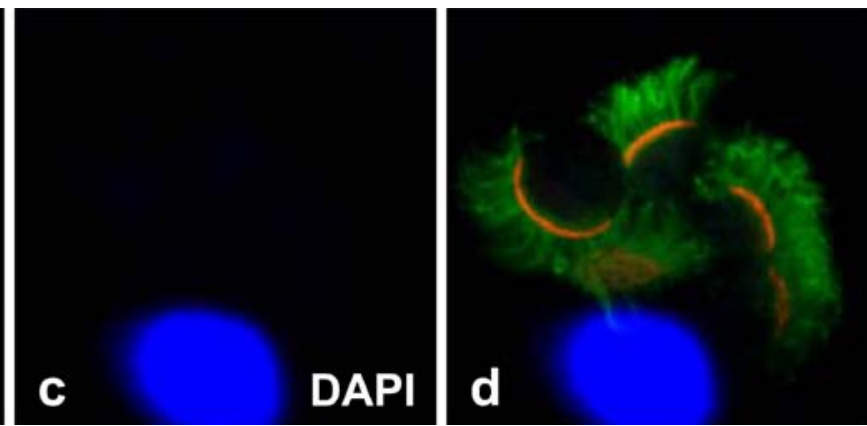

cilia and basal bodies thus verifying Odf 2 as a basal body protein. Nuclei were counterstained with DAPI (c). Merged image is presented in $\mathbf{d}$ 
interference contrast microscopy (Fig. 4d, e). Odf2 colocalized with acetylated tubulin in the OS and marked the connecting cilium as a dot (Fig. 4b, arrowheads in a, b). These results confirmed Odf2 as a component of the photoreceptor primary cilium. However, the colocalization with $\gamma$-tubulin in retinal cross sections additionally suggests localization to the centrosomes.

Importantly, mutations in genes that encode cilia components are common causes of disease, as might also be the case for retinal degeneration. Thus, an effort has recently been undertaken to identify the proteome of the mouse PSC complex (Liu et al. 2007). Identification of novel ciliary proteins previously not known as ciliary components opens up new directions for studies of cilia formation and dysfunction: in this case, for the study of retinopathies. Odf2 is one of the proteins identified in the proteome of the PSC complex (Liu et al. 2007). Our results have thus confirmed Odf2 as a protein of the photoreceptor cilium and suggest that a dysfunction of Odf2, albeit so far not taken into consideration, might be involved in retinal degeneration.

Odf2 localizes to the basal body of kidney primary cilia

Kidney tissue were processed and sectioned as described. Immunostaining with antibodies against acetylated tubulin (green in Fig. 5) marked primary cilia and basal bodies. Odf2 was found to highlight the base of the primary cilium (red in Fig. 5) thus supporting Odf2 as a basal body protein in kidney cells.

Odf2 is a component of basal bodies of nasal epithelial cells

The nasal cavity consists of two types of epithelium. The first is a typical respiratory epithelium lining most of the respiratory tract. The second is the olfactory epithelium found in the chemosensory area of the nose. The olfactory epithelium is the organ of smell. It is composed of olfactory receptor cells, supporting cells, and basal cells. Olfactory cells are bipolar nerve cells from which several cilia extend. These cilia are non-motile and act as receptors of smell. The typical respiratory epithelium is found throughout the length of the air passages of the respiratory tract and lines most of the cavity in the nose. It consists of multi-ciliated epithelial cells, amongst others.

To localize endogenous Odf2, we cultivated nasal epithelial cells from adult mice on coverslips and processed them for immunocytology. Cilia and basal bodies were identified by staining for acetylated tubulin (Fig. 6b), thus demonstrating unequivocally that Odf2 is located at the basal bodies (Fig. 6a).

Our results show, for the first time, the expression pattern of Odf2 in adult tissues. In sections of the cerebellum and of muscle tissue, the immunological staining revealed approximate colocalization with $\gamma$-tubulin spots suggesting a centrosomal localization. In retinal sections, Odf 2 was found to colocalize with $\gamma$-tubulin at the outer border of the ONL, again suggesting a centrosomal localization. Additionally, in isolated PSC complexes, Odf2 localized to the axoneme of the OS, thus confirming Odf2 as a protein component of the photoreceptor primary cilium complex. Immunocytology on nasal epithelial cells, which displayed numerous cilia combined and anchored by their basal bodies, definitely demonstrated that Odf2 was a basal body protein.

Together, our results have revealed the widespread expression of Odf2 and a general location to centrosomes and basal bodies. Moreover, our results suggest the possibility that Odf2 contributes to assorted ciliopathies, including retinopathies, but such research into these aspects awaits functional knockdown studies. As accessibility and sampling of nasal epithelial cells from patients is a noninvasive method, this might become a reliable tool for immunological diagnosis of cilia-associated diseases in the near future. We are currently in the process of testing this non-invasive method and comparing its performance with conventional nasal biopsies.

Acknowledgment We gratefully acknowledge the excellent technical assistance of Jens Bunzendahl and the animal and technical support provided by Prof. Dr. W. Engel and Prof. Dr. Heidi Hahn, Institute of Human Genetics, University of Göttingen, Germany. Our thanks are also extended to Dr. Kristine Henningfeld, University of Göttingen, for carefully reading the manuscript.

Open Access This article is distributed under the terms of the Creative Commons Attribution Noncommercial License which permits any noncommercial use, distribution, and reproduction in any medium, provided the original author(s) and source are credited.

\section{References}

Badano JL, Mitsuma N, Beales PL, Katsanis N (2006) The ciliopathies: an emerging class of human genetic disorders. Annu Rev Genomics Hum Genet 7:125-148

Brohmann H, Pinnecke S, Hoyer-Fender S (1997) Identification and characterization of new cDNAs encoding outer dense fiber proteins of rat sperm. J Biol Chem 272:10327-10332

Bornens M (2002) Centrosome composition and microtubule anchoring mechanisms. Curr Opin Cell Biol 14:25-34

Donkor FF, Mönnich M, Czirr E, Hollemann T, Hoyer-Fender S (2004) Outer dense fiber protein 2 (ODF2) is a self-interacting centrosomal protein with affinity for microtubules. J Cell Sci 117:4643-4651

Hoyer-Fender S, Petersen C, Brohmann H, Rhee K, Wolgemuth DJ (1998) Mouse Odf2 cDNAs consist of evolutionary conserved as well as highly variable sequences and encode outer dense fiber proteins of the sperm tail. Mol Reprod Dev 51:167-175

Hüber D, Hoyer-Fender S (2007) Alternative splicing of exon 3b gives rise to ODF2 and Cenexin. Cytogenet Genome Res 119:68-73 (doi:10.1159/000109621) 
Hüber D, Geisler S, Monecke S, Hoyer-Fender S (2008) Molecular dissection of ODF2/Cenexin revealed a short stretch of amino acids necessary for targeting to the centrosome and the primary cilium. Eur J Cell Biol 87:137-146

Ishikawa H, Kubo A, Tsukita S, Tsukita S (2005) Odf2-deficient mother centrioles lack distal/subdistal appendages and the ability to generate primary cilia. Nat Cell Biol 7:517-524

Lange BMH, Gull K (1995) A molecular marker for centriole maturation in the mammalian cell cycle. J Cell Biol 130:919927

Liu Q, Tan G, Levenkova N, Li T, Pugh EN Jr, Rux JJ, Speicher DW, Pierce EA (2007) The proteome of the mouse photoreceptor sensory cilium complex. Mol Cell Proteomics 6:1299-1317

Michaud EJ, Yoder BK (2006) The primary cilium in cell signaling and cancer. Cancer Res 66:6463-6467
Nakagawa Y, Yamane Y, Okanoue T, Tsukita S, Tsukita S (2001) Outer dense fiber 2 is a widespread centrosome scaffold component preferentially associated with mother centrioles: its identification from isolated centrosomes. Mol Biol Cell 12:1687-1697

Salmon NA, Reijo Pera RA, Xu EY (2006) A gene trap knockout of the abundant sperm tail protein, outer dense fiber 2, results in preimplantation lethality. Genesis 44:515-522

Schalles U, Shao X, Hoorn FA van der, Oko R (1998) Developmental expression of the $84-\mathrm{kDa}$ ODF sperm protein: localization to both the cortex and medulla of outer dense fibers and to the connecting piece. Dev Biol 199:250-260

Soung NK, Kang YH, Kim K, Kamijo K, Yoon H, Seong YS, Kuo YL, Miki T, Kim SR, Kuriyama R, Giam CZ, Ahn CH, Lee KS (2006) Requirement of hCenexin for proper mitotic functions of polo-like kinase 1 at the centrosomes. Mol Cell Biol 26:8316-8335 